\title{
Studies on Medicinal Resources from Livestock. I. Anti-allergic Effects of Pig Bile. (1) ${ }^{1)}$
}

\author{
Michinori Kubo, ${ }^{a}$ Tatsuyoshi NaKagami, ${ }^{*, b}$ Non Yamasaki, ${ }^{b}$ Masaaki Ito, ${ }^{a}$ Mari Nogami ${ }^{a}$ and Shiro TajI ${ }^{b}$ \\ Faculty of Pharmaceutical Sciences, Kinki University, ${ }^{a}$ 3-4-1, Kowakae, Higashiosaka, Osaka 577, Japan and R \& D Center, Nippon Meat Packers Inc. ${ }^{b}$ \\ 451, Takahata, Hiraoka-cho, Kakogawa, Hyogo 675-01, Japan. Received April 12, 1989
}

\begin{abstract}
Anti-allergic activities of animal biles and commercially available bile acids were evaluated in experimental allergic disease models. Pig bile exhibited marked preventive effects on the models of delayed-type hypersensitivity (type IV allergy), picryl chloride-induced contact dermatitis (PC-CD) and sheep red blood cells (SRBC)-induced footpad swelling in mice. Fel ursi (dried bear gallbladder) also had an inhibitory effect on PC-CD, whereas ox bile, chicken bile and bile acids had no effect on ether of the models.
\end{abstract}

Keywords anti-allergic effect; pig bile; delayed-type hypersensitivity; picryl chloride-induced contact dermatitis

Various animal organs have been widely used in traditional oriental medicine. "Bezoar bovis (bovine gallstone)" and "Fel ursi (bear gallbladder)" are representative examples. According to recent Chinese reports ${ }^{2,3)}$ on traditional Chinese medicine, animal biles can be used for therapy of bronchitis, asthma and various hypersensitivities. As regards pharmacological effects of animal biles, Aoki et $a l^{4)}$ reported that pig bile powder stimulated pancreatic juice secretion and bile flow in rats, promoted the passage of charcoal meal in the small intestine of mice, and produced an increase of liver blood flow in rabbits. The pharmacological effects of animal biles on bronchitis, asthma and hypersensitivities, however, have not yet been studied. Since these diseases are associated with various allergic reactions, we examined anti-allergic effects of animal biles in the present study.

Generally, allergic reactions fall into two categories, acute and delayed types. Furthermore, they can be divided into four or five types on the basis of the mechanisms of allergic diseases. For each type of allergy, experimental disease models have been recently developed. The models used in the present study were picryl chloride-induced contact dermatitis (PC-CD) and sheep red blood cells (SRBC)-induced footpad swelling in mice as delayed type hypersensitivities (type IV).

\section{Experimental}

Materials Biles of pigs (triple crosses among Large Yorkshire, Landrace, Duroc and Hampshire strains, and Berkshire strain), oxen (Holstein Friesian strain) and chickens (crosses between female White Plymouth Rock and male White Cornish strains) were collected from gallbladders with sterile plastic syringes, pooled and lyophilized. "Fel ursi" (dried bear gallbladder), a traditional crude drug, and bile acids (hyodeoxycholic acid (HDCA), sodium glycochenodeoxycholate (GCDCA), ursodeoxycholic acid, sodium taurochenodeoxycholate, glycocholic acid, and sodium tauroursodeoxycholate; Sigma Chem. Co.) were obtained commercially.

Animals Male Wistar rats, male ddY mice and female ICR mice were used. They were housed in an air-conditioined room with a commercial chow (Oriental Yeast Co., Ltd.) and tap water ad libitum.

Inhibitory Effect on PC-CD in Mice PC-CD in mice, an experimental model of delayed-type hypersensitivity, was induced according to the method of Asherson and Dtak. ${ }^{5}$ Male ddY mice weighing 18 to $22 \mathrm{~g}$ were sensitized by applying $0.1 \mathrm{ml}$ of $7 \% \mathrm{PC}$ in ethanol to their abdominal skins, which had been shaved on the previous day. After $7 \mathrm{~d}$, contact dermatitis was induced by applying $0.02 \mathrm{ml}$ of $1 \% \mathrm{PC}$ in olive oil to each ear lobe. After a further $3 \mathrm{~d}$, resensitization was performed in the same manner with $7 \% \mathrm{PC}$ in ethanol. Seven days later, contact dermatitis was induced again by $1 \% \mathrm{PC}$ in olive oil (challenge). Each test drug, suspended in distilled water, was orally administered immediately before and $16 \mathrm{~h}$ after challenge. Prednisolone (Sigma Chem. Co.,), a steroid drug, was used as a reference standard. Mice in the control group were orally given only distilled water in place of a drug suspension. Ear thickness was measured with a dial thickness gauge (Ozaki Co.,) immediately before $(B)$ and $24 \mathrm{~h}$. after challenge $(A)$. Ear swelling $(\%)$ was calculated from the mean value of $(A)$ and $(B)$ by using the following equation;

$$
\text { ear swelling }(\%)=(A / B-1) \times 100
$$

Inhibition rate $(\%)$ was calculated from ear swelling $(\%)$ of the control group $(C)$ and that of each test drug group $(D)$ by using the following equation;

$$
\text { inhibition }(\%)=(1-D / C) \times 100
$$

Immediately after the final measurement of ear swelling, the spleens of mice were removed to measure their wet weights.

Inhibitory Effect on SRBC-Induced Footpad Swelling in Mice Delayedtype hypersensitivity (DTH) was induced by SRBC according to the method of Kettman. ${ }^{6)}$ Female ICR mice were sensitized by the s.c. injection of $10^{8} \mathrm{SRBC} /$ mouse into their backs. After $4 \mathrm{~d}$, they were challenged by the i.c. injection of $10^{8} \mathrm{SRBC} /$ mouse into their right hind footpads. Each test drug, suspended in distilled water, was orally given immediately before and $16 \mathrm{~h}$ after challenge. Prednisolone was used as a reference standard. Mice in the control group were orally given only distilled water in place of a drug suspension. Footpad swelling was measured by the use of a dial thickness gauge (Ozaki Co.,) $24 \mathrm{~h}$ after challenge. Footpad swelling $(\%)$ was calculated by using the following equation;

footpad swelling $(\%)=(A / B-1) \times 100$

$A$ : Thickness of the right hind footpad $24 \mathrm{~h}$ after challenge

$B$ : Thickness of the left hind footpad $24 \mathrm{~h}$ after challenge

Calculation of inhibition ( $\%$ ) and measurement of spleen weight were done in the same manner as in the PC-CD test.

Chemical Analysis The total bile acid content was determined by the enzyme method. ${ }^{7)}$ The cholesterol content was determined by the $o$ phthalaldehyde method. ${ }^{8)}$ The fatty acid content was determined by the bathocupuroine method. ${ }^{9)}$ The phospholipid content was determined by the molybdenum blue method. ${ }^{10}$ The total neutral sugar content was determined by the phenol-sulfate method. ${ }^{11}$ The protein content was determined by the Lowry method ${ }^{12)}$ with bovine serum albumin as a standard protein.

Statistical Analysis All data are means \pm S.E. Statistical analysis was performed by using Student's $t$ test.

\section{Results and Discussion}

The inhibitory effects of the various animal biles on the models of DTH in mice were examined. Figure 1 indicates that pig bile remarkably inhibited the PC-induced ear swelling in mice at a dose of $100 \mathrm{mg} / \mathrm{kg}$. Bear bile also showed an inhibitory effect, whereas ox and chicken biles had no effect. Pig bile prepared from Berkshire strain was also effective, showing $34.0 \%$ inhibition at a dose of $100 \mathrm{mg} / \mathrm{kg}$ (data not shown). The effect on SRBC-induced footpad swelling in mice is shown in Fig. 2. Pig bile 


\begin{tabular}{|c|c|c|c|c|}
\hline \multirow[b]{2}{*}{ Treatment } & \multicolumn{2}{|r|}{ Ear swelling (\%) } & \multicolumn{2}{|r|}{ Spleen (\% b.w.) } \\
\hline & $(\mathrm{mg} / \mathrm{kg})$ & $\begin{array}{lll}0 & 50 & 100 \\
\end{array}$ & Inhibition $(\%)$ & $0 \quad 0.5$ \\
\hline Control & - & 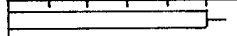 & - & - \\
\hline Pig bile & 20 & $\square-$ & 30.8 & $\square$ \\
\hline F ig one & 100 & $\square-(b)$ & 60.7 & $\square$ \\
\hline Chicken bile & 20 & $\longrightarrow$ & 12.2 & $\Longrightarrow$ \\
\hline & 100 & $\Longrightarrow$ & 18.0 & $\square$ \\
\hline Ox bile & 20 & $\longrightarrow$ & 26.0 & 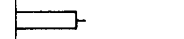 \\
\hline & 100 & $\Longrightarrow$ & 13.9 & ב- \\
\hline Bear bile & 20 & $\longrightarrow-a_{1}$ & 36.8 & $\Longrightarrow$ \\
\hline & 100 & $\square^{a)}$ & 31.7 & $\square$ \\
\hline Prednisolone & 20 & $\square^{b 1}$ & 51.2 & $\square-b^{b}$ \\
\hline
\end{tabular}

Fig. 1. Effects of Biles Prepared from Various Species of Animals on PC-CD in Mice

Each group includes 10 animals. Values are means \pm S.E. Significantly different from the control group at $a$ ) $p<0.05, b) p<0.01$. exhibited an inhibitory effect at a dose of $500 \mathrm{mg} / \mathrm{kg}$, whereas ox and chicken biles had no effect, as in the case of PC-CD. Pig bile from Berkshire strain had no significant effect in this experiment (data not shown).

Animal biles contain bile acids, bile pigment, lipids and glycoproteins as major solid components. The result of chemical analysis of the lyophilized pig bile is shown in Table I. Namba et al. ${ }^{13)}$ reported that glycohyodeoxycholic acid and glycochenodeoxycholic acid made up approximately $90 \%$ of bile acids in pig bile. Aoki et al. $^{14)}$ also reported that HDCA and chenodeoxycholic acid were main components of pig bile powder. In this study, six kinds of commercially available bile acids including HDCA and GCDCA were examined for inhibitory effects on the models of DTH to find the active components of pig bile.

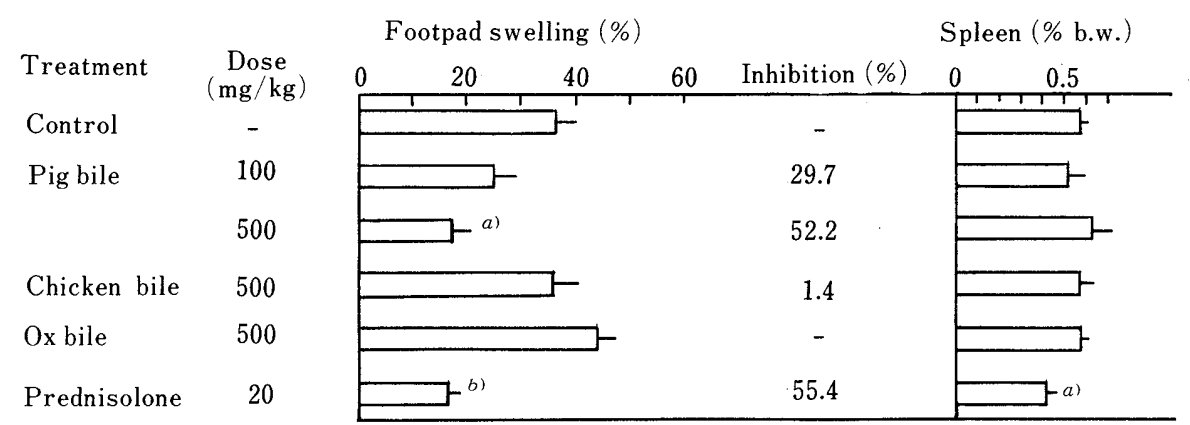

Fig. 2. Effects of Biles Prepared from Various Species of Animals on SRBC-DTH in Mice

Each group includes 10 animals. Values are means \pm S.E. Significantly different from the control group at $a) p<0.05, b) p<0.01$.

\begin{tabular}{|c|c|c|c|c|c|}
\hline \multirow{2}{*}{ Treatment } & \multicolumn{3}{|c|}{ Ear swelling (\%) } & \multicolumn{2}{|c|}{ Spleen (\% b.w. } \\
\hline & $\begin{array}{l}\text { Dose } \\
\text { (mg/kg) }\end{array}$ & 50 & 100 & Inhibition (\%) & $0 \quad 0.5$ \\
\hline Control & - & & & - & $\longrightarrow$ \\
\hline HDCA & 20 & & - & 1.1 & $\square$ \\
\hline GCDCA & 20 & & - & 2.7 & $\square$ \\
\hline UDCA & 20 & 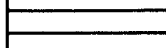 & - & 6.4 & $\longrightarrow$ \\
\hline TCDCA & 20 & & 工- & - & $\square$ \\
\hline $\mathrm{GCA}$ & 20 & & ᄂ & - & $\square$ \\
\hline TUDCA & 20 & 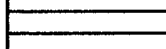 & & 3.5 & $\square$ \\
\hline Prednisolone & 20 & 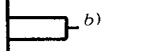 & & 74.7 & $\square^{a)}$ \\
\hline
\end{tabular}

Fig. 3. Effects of Some Bile Acids on PC-CD in Mice

Each group includes 10 animals. Values are means \pm S.E. Significantly different from the control group at $a$ ) $p<0.05, b) p<0.01$.

HDCA, hyodeoxycholic acid; GCDCA, sodium glycochenodeoxycholate; UDCA, ursodeoxycholic acid; TCDCA, sodium taurochenodeoxycholate; GCA, glycocholic acid; TUDCA, sodium tauroursodeoxycholate.

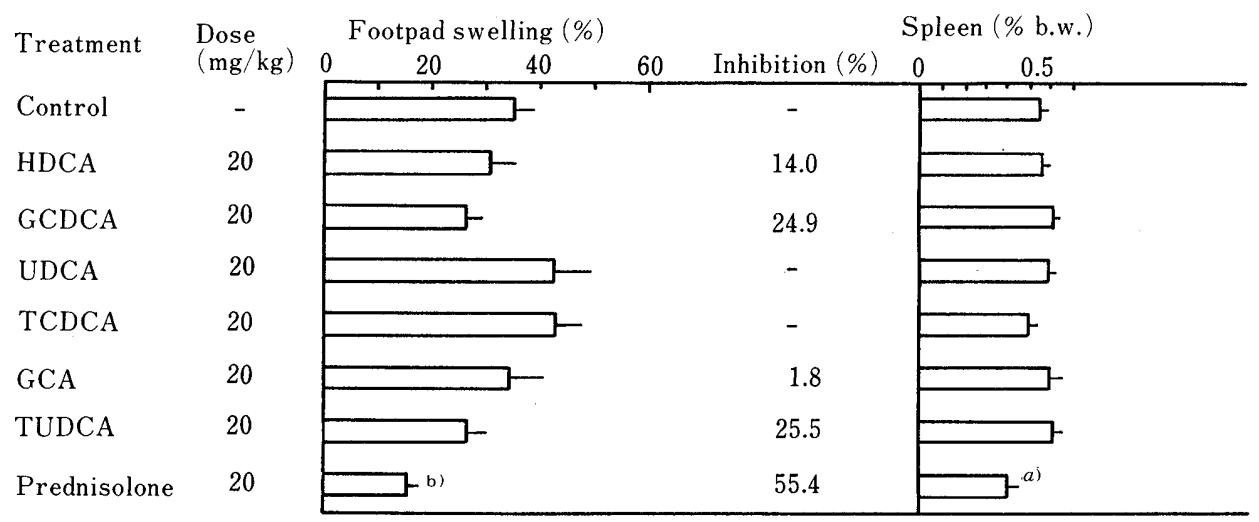

Fig. 4. Effects of Some Bile Acids on SRBC-DTH in Mice

Each group includes 10 animals. Values are means \pm S.E. Significantly different from the control group at $a$ ) $p<0.05, b) p<0.01$. 
TABLE I. Analysis of Lyophilized Pig Bile

\begin{tabular}{lr}
\hline \hline & $\mathrm{mg} / \mathrm{g}$ \\
\hline Total bile acid $^{a)}$ & 572 \\
Cholesterol $^{b)}$ & 25 \\
Fatty acid $^{()}$ & 80 \\
Phospholipid $^{()}$ & 6 \\
Total sugar $^{()}$ & 52 \\
Protein $^{\text {) }}$ & 48
\end{tabular}

a) Enzyme method. b) $o$-Phthalaldehyde method. c) Bathocupuroine method. d) Molybdenum blue method. e) Phenol-sulfate method. $f$ ) Lowry method.

However, those bile acids had no effect on either of the models at a dose of $20 \mathrm{mg} / \mathrm{kg}$ (Figs. 3 and 4). The dose of $20 \mathrm{mg}$ each bile acid $/ \mathrm{kg}$ is equivalent to that of $100-500 \mathrm{mg}$ or more of pig bile powder $/ \mathrm{kg}$ on the basis of each bile acid content in pig bile powder according to the study of Aoki et $a l .{ }^{14)}$ The above findings suggest that the active components of pig bile are not bile acids but some other species-specific substances (e.g. glycoproteins).

Prednisolone, a reference standard, exhibited a strong preventive effect on PC-CD and SRBC-induced footpad swelling at a dose of $20 \mathrm{mg} / \mathrm{kg}$. It was also shown that treatment with prednisolone significantly reduced the spleen weight of mice (Figs. 1, 2, 3 and 4).

Aoki et al. ${ }^{15)}$ reported that acute and chronic toxicities of pig bile powder were very low. In the present study, pig bile showed no serious side effect such as the splenic atrophy caused by steroid drugs (e.g. prednisolone). It is remarkable that pig bile produces a potent inhibitory effect on DTH without apparent adverse effect.

Further studies are in progress on the active components in pig bile and on the mode of action.

\section{References and Notes}

1) Part of this paper was presented at the 106th Annual Meeting of the Pharmaceutical Society of Japan, Chiba, April 1986.

2) S. Gao, D. Dai, Q. Fan and M. Deng, "Chag Jian Yao Yong Dong Wu (常見薬用動物), " Shanghai Ke Xue Ji Shu Publisher, Shanghai, 1984 , p. 265.

3) J. Ji and Y. Zhao, "Shandong Yao Yong Wu (山東薬用動物)," Shandong Ke Xue Ji Shu Publisher, Jinan, 1979, p. 431.

4) K. Aoki, Y. Ishiguro, Y. Fujimoto, S. Kondo, K. Tachibana and T. Nakajima, Oyoyakuri, 18, 363 (1979).

5) G. L. Asherson and W. Dtak, Immunology, 15, 405 (1968).

6) J. R. Kettman, Immunopharmacology, 1, 21 (1978).

7) F. Mashige, Clin. Chem., 27, 1352 (1981)

8) A. Zlatkis and B. Zak, Anal. Biochem., 29, 143 (1969).

9) W. G. Duncombe, Clin. Chem. Acta, 9, 122 (1964).

10) W. R. Bloor, J. Biol. Chem., 82, 273 (1929).

11) M. Dubois, Anal. Chem., 28, 350 (1956).

12) O. H. Lowry, J. Biol. Chem., 193, 265 (1951).

13) T. Namba, S. Nunome, M. Hattari, S. Higasidate and T. Maekubo, Yakugaku Zasshi, 102, 760 (1982).

14) K. Aoki, Y. Ito, Y. Fujimoto, S. Kondo, K. Tachibana and Y. Kobayashi, Oyoyakuri, 14, 815 (1977).

15) K. Aoki, Y. Fujimoto, S. Kondo, K. Tachibana and T. Nakajima, Oyoyakuri, 19, 901 (1980). 